\title{
TECNOLOGÍA ÓSEA EN EL OESTE DE LA REGIÓN PAMPEANA: IDENTIFICACIÓN DE LAS TÉCNICAS DE MANUFACTURA A PARTIR DE EVIDENCIAS ARQUEOLÓGICAS Y EXPERIMENTALES
}

\author{
BONE TECHNOLOGY IN THE WESTERN PAMPEAN REGION: \\ IDENTIFYING MANUFACTURING TECHNIQUES FROM \\ ARCHAEOLOGICAL AND EXPERIMENTAL EVIDENCE
}

\author{
María Clara Álvarez ${ }^{1}$
}

\begin{abstract}
En este trabajo se presentan los resultados del estudio de huesos vinculados con la tecnología, provenientes del sitio arqueológico Laguna de los Pampas (LLP), localizado en la subregión Pampa Húmeda de Argentina. Los especímenes fueron estudiados aplicando el enfoque tecnológico al análisis de los instrumentos óseos por secuencias de operaciones, en el sentido de cadenas operativas de la manufactura. El objetivo fue identificar el proceso de manufactura de las formas base en el sitio LLP, a través del estudio de los restos arqueológicos, así como de trabajos experimentales. Se reconstruyó la cadena operativa teórica para la manufactura de las formas base en tibias y metapodios de guanaco. En general, los resultados indican que los instrumentos óseos habrían sido confeccionados en el sitio para el caso de la tibia y que este elemento fue seleccionado como núcleo para la obtención de astillas.
\end{abstract}

Palabras claves: manufactura ósea, cadena operativa, pampa húmeda, área Oeste, Lama guanicoe.

This paper presents the results of a study of bone specimens related to bone technology from the Laguna de los Pampas (LLP) archaeological site, located in the Humid Pampean subregion in Argentina. The bone tools were analyzed using a technological approach examining sequences of operations, that is, chaînes opératoires of manufacture. The objective was to identify the manufacturing process of bone tools at the LLP site by analyzing the archaeological remains and through experimental studies. The hypothetical sequences for manufacturing bone tools were reconstructed using the tibia and metapodial bones of guanaco, a native camelid. The results indicate that tibia bone tools were crafted at the LLP site, and that this particular bone was favored as a core for the production of shaft splinters.

Key words: Bone manufacture, chaîne opératoire, Humid Pampa, western Pampean region, Lama guanicoe.

Los análisis de la tecnología ósea de los grupos cazadores-recolectores se han incrementado en los últimos años en Argentina, particularmente en algunas regiones, como es el caso de la Patagonia (Borella y Buc 2009; Borrero y Borella 2010; Hajduk y Lezcano 2005; Orquera y Piana 1999; Paunero et al. 2010; Scheinsohn 2010; entre otros) y el área Nordeste (Bonomo et al. 2011; Pérez Jimeno 2007), donde existe una larga tradición de análisis de este tipo de registro (Caggiano 1984; Lothrop 1932; Rodríguez 2001; Serrano 1950; Torres 1911; entre otros). En el caso de la región pampeana ${ }^{1}$, las investigaciones sobre tecnología ósea se han centrado en el área Norte, para la cual se cuenta con importantes evidencias durante el Holoceno tardío (Acosta 2005; Bonomo 2013; Bonomo et al. 2009; Buc 2011; Buc y Silvestre 2006; Buc y Pérez Jimeno 2010; Loponte y Buc 2012; Pérez Jimeno 2004; Pérez Jimeno et al. 2010; Politis et al. 2011; entre otros). En menor medida, estos estudios han sido llevados a cabo en la Depresión del Salado (González 2005; González et al. 2004), las Sierras de Tandilia (Mazzanti y Valverde 2001), el área Interserrana (Johnson et al. 2000; Messineo y Pal 2011) y recientemente el Oeste (Cornaglia Fernández y Buc 2013; Politis et al. 2012). El escaso desarrollo de los análisis de tecnología ósea en algunas de las áreas se debe a que estos materiales son exiguos y presentan modificaciones mínimas del hueso, lo cual llevó a un sesgo en su estudio. En este

1 CONICET-INCUAPA (Investigaciones Arqueológicas y Paleontológicas del Cuaternario Pampeano), Facultad de Ciencias Sociales, Universidad Nacional del Centro de la Provincia de Buenos Aires, Avenida del Valle 5737, B7400JWI Olavarría, Buenos Aires, Argentina. malvarez@soc.unicen.edu.ar 
sentido, entendiendo a los artefactos como parte de un continuo en la manufactura, Choyke (1997) distinguió dos clases. La Clase 1 está representada por instrumentos planificados en estadíos múltiples, que tienden a ser producidos de materias primas seleccionadas y tienen mayor inversión de trabajo. La Clase 2 está constituida por instrumentos realizados sobre especímenes que se fracturaron en una forma azarosa y no tienden a ser conservados (Choyke 1997).

En este trabajo se presenta el análisis de los especímenes óseos con evidencias de formatización antrópica procedentes del sitio arqueológico Laguna de los Pampas (LLP), localizado en el Oeste de la región pampeana argentina (Figura 1). El análisis de estos restos con trazas de manufactura y/o uso permitió incrementar el conocimiento de la tecnología ósea para esta área. En la misma, el único antecedente disponible sobre este tema es un trabajo de reciente publicación (Cornaglia Fernández y Buc 2013). En esta oportunidad, los especímenes estudiados están vinculados con la Clase 1 (Choyke 1997) y presentan características que, como se verá más adelante, permitieron inferir el uso de la técnica de splinter (Newcomer 1974; Yesner y Bonnichsen 1979). Esta consiste en el raspado y pulido del hueso para su limpieza y preparación y el trazado de surcos longitudinales a las diáfisis de los huesos largos (ranurado), con el objetivo de separar controladamente porciones del elemento. Con el mismo sentido se realiza el marcado o aserrado perimetral en las epífisis, las cuales suelen constituir los desechos de esta secuencia de pasos (Campana 1980; Choyke 1997).

Los especímenes fueron estudiados aplicando el enfoque tecnológico al análisis de los instrumentos óseos, como fue propuesto por David (2007), incluyendo no solo los instrumentos, sino también los desechos de la manufactura, así como cualquier otra evidencia del proceso de producción. De esta manera, la identificación de las técnicas utilizadas en la formatización permite la reconstrucción teórica de los métodos de manufactura por secuencias de operaciones en la forma de cadenas operativas (Pelegrin et al. 1988). Lemmonier (1976) postula que las actividades técnicas pueden ser analizadas a partir de (1) los objetos (p.ej., instrumentos, productos de talla), (2) los gestos o las operaciones que estudian los procesos técnicos (p.ej., talla experimental) y (3) los conocimientos específicos. Este análisis permite registrar el esquema conceptual preexistente a todo proyecto del tallador y paralelamente, el espacio de posibilidades y de elecciones que posee según sus conocimientos, sus tradiciones culturales, sus recursos y los límites que le impongan las materias primas.

Los análisis macroscópicos de la tecnología ósea con el objetivo de identificar técnicas de manufactura se han desarrollado en diversas regiones del mundo (Camps-Fabrer y D'Anna 1977; David 2007; Emery 2008; Newcomer 1974; Olsen 1980; Pickering 1980; Russell 2006; Yesner y Bonnichsen 1979; entre otros). Sin embargo, en la región pampeana argentina los estudios de este tipo son escasos, con algunas excepciones (p.ej., Bonomo et al. 2009; Loponte y Buc 2012; Mazzanti y Valverde 2001). Considerando esto, el objetivo de este trabajo es identificar el proceso de obtención de las formas base en el sitio LLP a través del estudio de los restos arqueológicos, junto con el desarrollo de estudios experimentales, y de esta forma evaluar si los pasos iniciales de la producción de instrumentos fueron realizados en el sitio o en otros lugares. El concepto de cadena operativa es utilizado como una herramienta interpretativa para entender cómo las prácticas tecnológicas de la vida cotidiana integran a los distintos agentes dentro de una dimensión social colectiva. En este trabajo solo se presenta parte de los resultados de los estudios de las técnicas que fueron utilizadas para la fabricación de instrumentos óseos en un sitio particular de la región pampeana. Sin embargo, el objetivo general se enmarca en una escala más amplia, para lo cual será necesario analizar más casos de estudio para poder dar cuenta de las acciones humanas que modificaron la materia prima. En este sentido, a largo plazo se necesita más información acerca de los diferentes contextos donde los instrumentos óseos se encuentran, para lograr analizar la transformación de los mismos en bienes culturales. Asimismo, se deben incorporar estudios de grupos cazadores recolectores que brinden información acerca de la explotación faunística, la selección de materia prima ósea, entre otros. Finalmente, se requiere incorporar más experimentaciones, para profundizar en el estudio de las técnicas, así como conocer los comportamientos de los distintos huesos.

\section{Sitio Laguna de los Pampas}

El sitio LLP se localiza a lo largo del borde de la laguna homónima, en el sudeste del partido de Lincoln, provincia de Buenos Aires, Argentina 


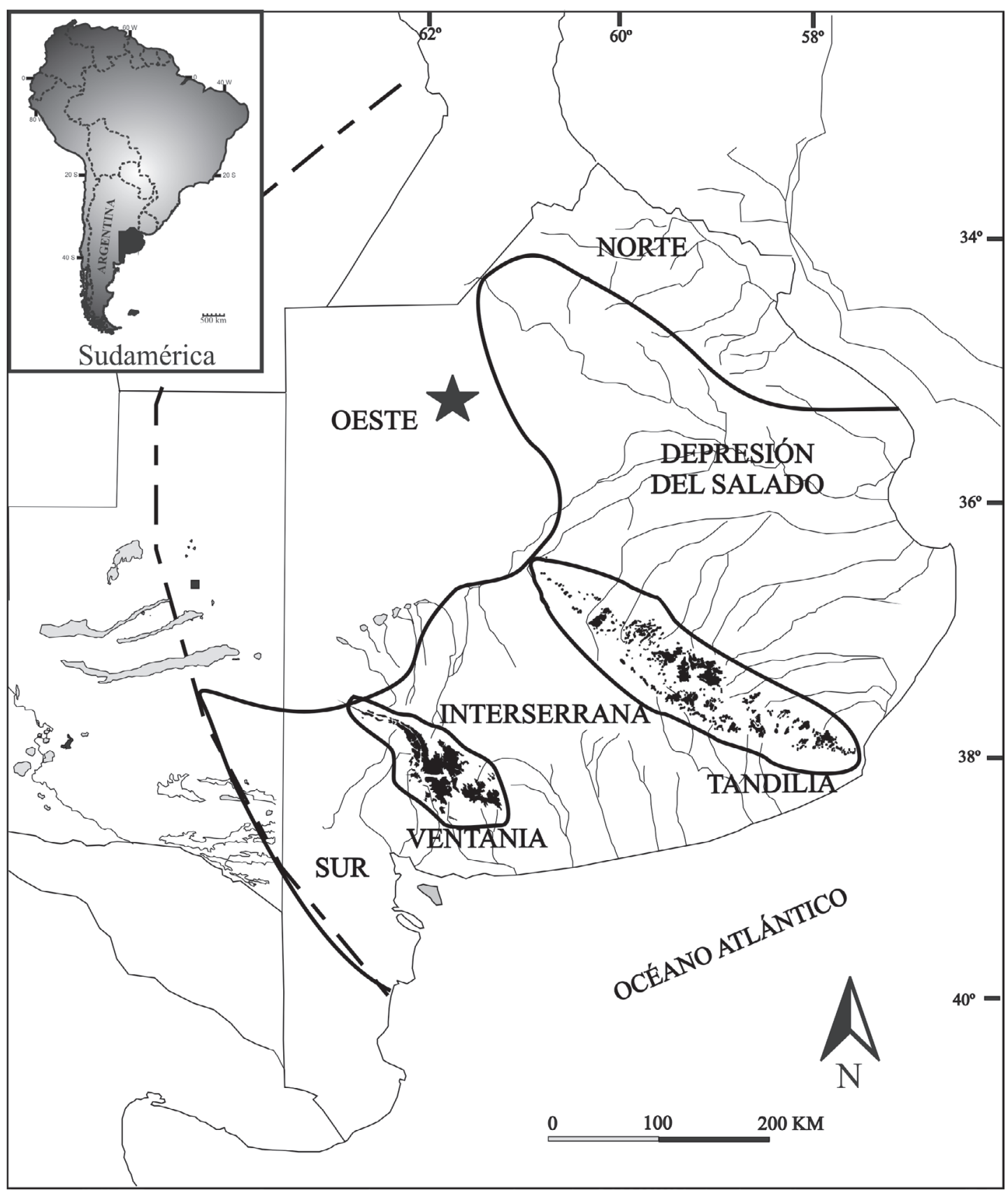

Figura 1. Delimitación de las áreas de la Pampa Húmeda (sensu Politis 1988) y ubicación del sitio Laguna de los Pampas (LLP, señalizado con una estrella).

Demarcation of the Humid Pampa areas (sensu Politis 1988) and location of Laguna de los Pampas archaeological site (LLP, indicated by a star).

(Figura 1). En el año 2008 la dinámica del agua causó la erosión de las barrancas por la línea de costa más alta y distintos materiales arqueológicos, como líticos, cerámica, restos óseos humanos y arqueofaunísticos, se expusieron en los bordes de la laguna. Los restos estaban distribuidos a lo largo de dos sectores de la costa; el Sector 1 (S35 $19^{\prime} 42^{\prime \prime}$ y W61 $\left.31^{\prime} 50^{\prime \prime}\right)$ y el Sector 2 (S35 ${ }^{\circ} 19^{\prime} 56^{\prime \prime}$ y W61 $31^{\circ} 53$ "). En el Sector 2 se recuperaron materiales arqueológicos dispersos, 
incluyendo huesos humanos (NMI=4), los cuales arrojaron dataciones correspondientes al Holoceno temprano (8.971 \pm 77 años radiocarbónicos a.p., AA-90127 y $8.835 \pm 83$ años radiocarbónicos a.p., AA-93221; Politis et al. 2012). En el Sector 1 se hallaron elementos esqueletarios humanos de cinco individuos, junto con otros materiales arqueológicos dispersos a lo largo de las márgenes de la laguna. Estos incluyen los artefactos óseos presentados en este trabajo. Entre febrero y marzo de 2009, los Drs. Politis y Messineo llevaron a cabo tareas de rescate en el área y todos los materiales de superficie fueron recolectados de este sector. Se realizaron 14 transectas de 10 metros de ancho cada una, con longitudes variables. Un fragmento de tibia de guanaco (Lama guanicoe) con evidencias tecnológicas fue datado en $5.684 \pm 61$ años radiocarbónicos a.p. (AA-93220; Politis et al. 2012), indicando que este sector de la laguna fue ocupado al menos durante el Holoceno Medio.

Los análisis de los materiales indicaron que en el área se asentaron grupos de cazadores-recolectores en diferentes momentos del Holoceno. Estas sociedades explotaron recurrentemente al guanaco para su subsistencia. La tecnología estuvo caracterizada por el uso de un amplio rango de materias primas líticas (principalmente ortocuarcita y ftanita) e incluyó artefactos unifaciales, así como morteros y manos. El sitio también fue utilizado para llevar a cabo prácticas mortuorias, al menos durante el Holoceno Temprano (Politis et al. 2012).

\section{Metodología}

Para el análisis de los restos óseos con evidencias tecnológicas $(\mathrm{N}=31)$ se realizó la identificación taxonómica y anatómica de cada espécimen, considerando además la porción del elemento, lateralidad, estado de fusión y edad estimada. Además, se relevó la presencia y frecuencia de las siguientes variables tafonómicas: meteorización, marcas de roedores, marcas de carnívoros, marcas de raíces, manganeso, pisoteo, tipo de fractura y abrasión sedimentaria (Behrensmeyer 1978; Binford 1981; Grayson 1984; Gutiérrez y Kaufmann 2007; Haynes 1980; Johnson 1985; Lyman 1994; Olsen y Shipman 1988; Shipman 1981). En primera instancia, las modificaciones de los especímenes fueron evaluadas considerando si su origen correspondía a causas antrópicas o naturales. Desde hace varias décadas existen diversos criterios que permiten identificar a uno u otro agente de acuerdo con las trazas generadas por los mismos (Johnson 1985; Haynes 2000; Lyman 1994; Shipman 1981).

Todos los materiales fueron analizados macroscópicamente, así como con lupa binocular (Iroscope) de 40X y con un microscopio digital (Dino-Lite AM413T) de hasta 200X, utilizando una luz incidente por medio de un iluminador de fibra óptica. Se consideraron los criterios macroscópicos propuestos por Johnson (1985) para la identificación de evidencias de manufactura ósea en los especímenes analizados, en relación con el aspecto de la superficie cortical de los huesos. Estos son los macrolascados, representados por grandes negativos producto del retoque intencional; y los microlascados, que son pequeños negativos generados por el uso y el pulido diferencial (Johnson 1985). Otros criterios utilizados a lo largo del trabajo son descriptos a continuación, con el objeto de evitar ambigüedades en su significado. Estas definiciones se encuentran en Borao Álvarez (2013:133), quien retoma y resume los trabajos de distintos autores (Averbouh 2000; Goutas 2004; Provenzano 2004; Tejero 2010). El raspado es la técnica que permite la eliminación de partículas sobre la materia, creando estrías agrupadas en bandas, paralelas y longitudinales al eje. El pulido refiere a una técnica que elimina micropartículas de la materia mediante la frotación de un agente abrasivo de grano muy fino. Las estrías que lo identifican son rectas o circulares organizadas en bandas planas y lisas. El ranurado indica la eliminación de partículas de materia mediante un movimiento unidireccional sucesivo que crea un surco largo y profundo con estrías paralelas al eje longitudinal y sección en forma de $\mathrm{u}$ (Borao Álvarez 2013:133).

Otro punto que es necesario aclarar es la diferencia entre marcado y aserrado perimetral (Acosta 2000). El marcado perimetral se realiza por medio de la percusión del hueso a lo largo de su circunferencia. De esta forma se obtiene una fractura en ángulo recto con respecto al eje longitudinal del hueso, en cuyo borde pueden observarse lascados (Muñoz y Belardi 1998). Por otra parte, el aserrado perimetral consiste en el seccionamiento alrededor de la diáfisis o epífisis, a través de sucesivos cortes, por medio de los cuales se llega hasta el canal medular (Acosta 2000).

En los casos en que fue posible, se utilizaron los criterios propuestos por David $(2004,2007)$ para la identificación de las técnicas de fractura, como 
por ejemplo preparada, por flexión o por splinter. Respecto de la clasificación de los instrumentos óseos terminados, se consideraron los grupos morfológicos piezas aguzadas (puntas), piezas con extremos biselados (biseles) y puntas cuyas facetas son obtusas (puntas romas). Los mismos fueron seleccionados de aquellos propuestos por Scheinsohn (2010) a partir de la morfología de la extremidad activa.

Se replicaron los métodos de manufactura inferidos a través de la observación del registro arqueológico. Para esto se realizaron siete experimentos que se detallan en los resultados (Tabla 1), utilizando elementos de guanaco y llama en estado seco y fresco. En el caso de los elementos secos se incluyeron unidades anatómicas sin meteorización, cuya médula ósea se encontraba seca, con una mayor dureza y menor flexibilidad que aquellos en estado fresco, recuperados en momentos perimortem, los cuales contenían médula ósea en buen estado. Todos los elementos fueron recolectados en la provincia de Río Negro, Argentina (Kaufmann 2009), con excepción del de llama, obtenido en un zoológico local (parque la Máxima, Olavarría).

El guanaco no habita actualmente la subregión Pampa Húmeda, con excepción de un parque provincial localizado en el área de Ventania. Dado que las carcasas de este ungulado no están disponibles localmente, se utilizaron mayoritariamente huesos secos para los experimentos. La desventaja de estos en relación con aquellos que se encuentran en estado húmedo es que las huellas obtenidas mediante la manufactura tienden a presentar microfracturas y lascados en lugar de ser cortes netos (Acosta et al. 2011). Por este motivo se incluyó el elemento de llama, utilizado en la experimentación debido a sus características osteológicas y morfológicas, similares a las del guanaco (Pacheco et al. 1979).

Las materias primas líticas empleadas en los experimentos provienen de las sierras Diamante y Bayas (área de Tandilia). Se utilizaron raspadores, lascas con filos naturales, puntas buriladas, pièces esquillées (sensu Demars y Laurent 1989), percutores (de asta, granito y cuarcita) y un yunque (cuarcita). Los experimentos fueron llevados a cabo en el campus de la Facultad de Ciencias Sociales (UNICEN), provincia de Buenos Aires, Olavarría. Se registró el tiempo transcurrido desde el inicio de la manufactura (incluyendo el tallado de artefactos líticos) hasta la obtención de las formas base. Posteriormente se llevó a cabo la elaboración de instrumentos óseos, similares a los recuperados en el registro arqueológico, tareas que no son descriptas en esta oportunidad.

\section{Resultados}

\section{Aspectos generales de la muestra}

Se analizó el conjunto total de restos faunísticos del Sector 1 de LLP $(\mathrm{N}=1.357)$. Las especies identificadas fueron guanaco, venado de las pampas (Ozotoceros bezoarticus), ñandú (Rhea americana), peludo (Chaetophractus villosus), zorrino (Conepatus sp.), puma (Puma concolor), vizcacha (Lagostomus maximus), comadreja overa (Didelphis albiventris), megamamígeros (Scelidoterium sp.) y roedores caviomorfos (Galea sp. y Ctenomys sp.) (ver detalles en Politis et al. 2012). Los elementos de guanaco son ampliamente predominantes en la muestra en relación con los de animales de menor tamaño. Del total de huesos identificados en LLP, las únicas especies con evidencias de procesamiento antrópico son: el guanaco (NISP=1181; $\mathrm{MNI}=37$ ), el venado de las pampas (NISP $=64 ; \mathrm{MNI}=6$ ) y el ñandú (NISP=23; MNI=4). Solo el ca. 2\% de los especímenes presenta huellas de corte, pero hay una importante frecuencia de fracturas de origen antrópico (ca. 22\%) (Politis et al. 2012).

Los análisis tafonómicos indicaron la presencia de agentes destructivos, como roedores (ca. 74\%) y acción de raíces (ca. 36\%). También se relevó la depositación de carbonato de calcio $\left(\mathrm{CaCO}_{3}\right)$ en una proporción significativa de la muestra (ca. 49\%), lo cual también contribuyó a la preservación de la superficie de los restos, como fue observado para otros sitios de la región pampeana (Gutiérrez 2006).

\section{Evidencias arqueológicas de la tecnología ósea}

Se determinó la presencia de 31 restos óseos con evidencias tecnológicas descritos en la Tabla 2. De estos, 15 correspondían a desechos de la manufactura y nueve a fragmentos de diáfisis que presentaban modificaciones y fueron determinados como fragmentos de formas base, o bien de instrumentos. Finalmente, se registraron siete instrumentos que conservaban sus extremidades activas completas y pudieron catalogarse como terminados. Se identificaron los siguientes taxones: 26 especímentes de la especie guanaco (22 tibias, un metatarso, un metacarpo, un radiocúbito y un fémur), uno de 


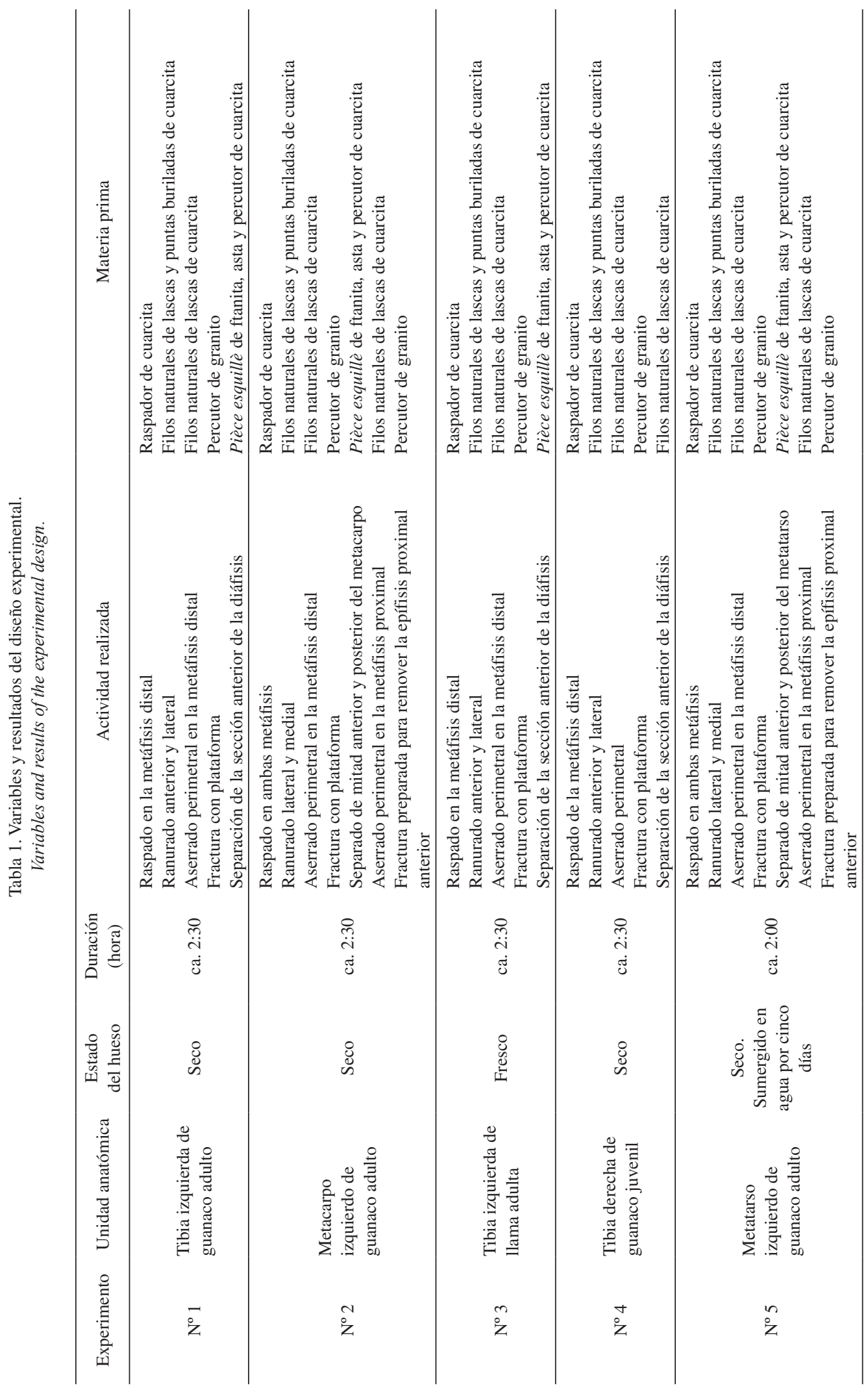


ñandú (tarsometatarso) y cuatro fueron huesos largos no identificados correspondientes a vertebrados o artiodáctilos, posiblemente guanacos. En todos los casos en que la edad pudo ser estimada los restos correspondían a individuos adultos.

En relación con los instrumentos terminados, cuatro de ellos son biseles (L.LLP.S1.2374, Figura 2a; L.LLP.S1.619, Figura 2b; L.LLP.S1.622, Figura 2c; L.LLP.S1.1429, Figura 2d). Este último es el único instrumento manufacturado sobre un elemento de ñandú. La pieza L.LLP.S1.1448 (Figura 2e) corresponde a un fragmento de instrumento que no pudo ser asignado a un grupo morfológico. También se identificó una pieza (L.LLP.S1.2697, Figura 2f) que corresponde al grupo morfológico punta, pero debido a las características de la misma podría describirse en mayor detalle como una punta de proyectil apedunculada. En el borde derecho se identificaron microlascados y en su cara anterior un adelgazamiento, los cuales podrían haber sido producidos por presión y talla por percusión indirecta, respectivamente. Finalmente, el instrumento L.LLP.S1.703 (Figura 2g) corresponde a una punta roma, que presenta estrías, las cuales no tienen una orientación definida.

En el caso de los desechos de manufactura (Figura 3; Tabla 2), cuatro de ellos corresponden a epífisis proximales de huesos largos de guanaco (un metacarpo, un metatarso y dos tibias) y 11 a epífisis distales (10 tibias y un fémur). Uno de los fragmentos de tibia distal de guanaco fue aquel datado en ca. 5700 años a.p. Todos estos restos presentaban una o más de las siguientes características: raspado, ranurado y marcado o aserrado perimetral. En el caso de las tibias, el ranurado de las epífisis distales se localiza en las caras anterior y lateral de las mismas y la mayoría de ellas presentan marcado $(n=7)$ o aserrado perimetral $(n=2)$. Por otro lado, los dos especímenes de metapodios corresponden a la mitad anterior de la epífisis y presentan ranurado en los lados medial y lateral y aserrado perimetral.

Se identificaron nueve fragmentos de diáfisis con modificaciones antrópicas, los cuales podrían corresponder a fragmentos de formas base o instrumentos fracturados. Siete de estos corresponden a guanaco (seis tibias y un radiocúbito) y dos a artiodáctilo (huesos largos indeterminados). Todos estos restos de fragmentos de diáfisis presentan pulido, raspado, surcos y/o remanentes de marcado o aserrado perimetral (Figura 4; Tabla 2). 


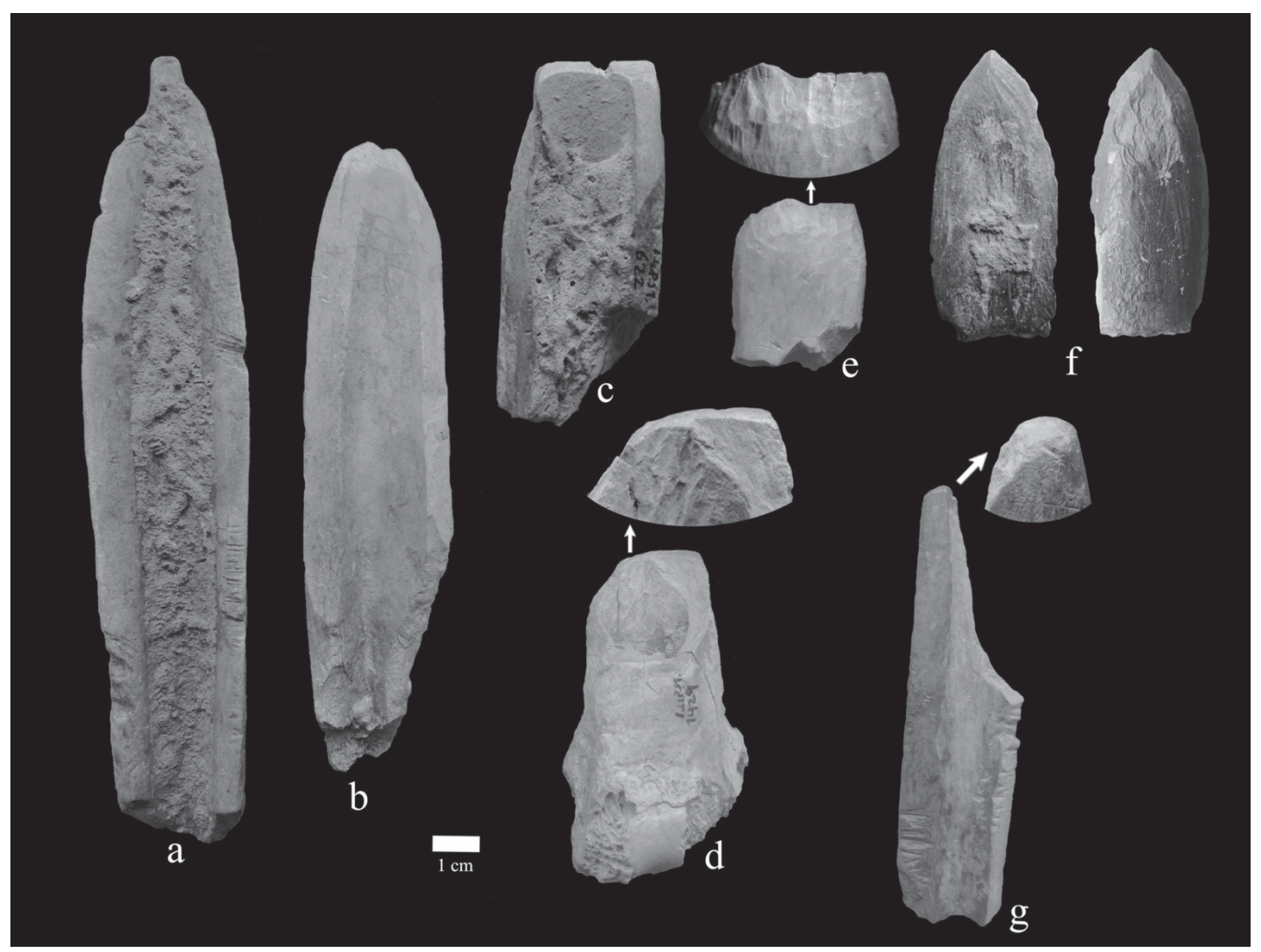

Figura 2. (a-c) Bisel sobre tibia de guanaco; (d) bisel sobre tibio-tarso de ñandú; (e) grupo morfológico indeterminado sobre elemento de artiodáctilo; (f) vista dorsal y ventral de punta manufacturada sobre elemento de artiodáctilo; $(\mathrm{g}$ ) punta roma sobre tibia de guanaco.

(a-c) Bevelled end of a tibia of a guanaco; $(d)$ bevelled end of a tarsal-metatarsal of a greater rhea; (e) unidentified morphological group of artiodactyl element; $(f)$ dorsal and ventral views of a point on an artiodactyl bone; $(g)$ blunt point on a tibia of a guanaco.

\section{Diseño experimental}

En este apartado solo se detallan algunas observaciones generales obtenidas a partir del desarrollo de las experimentaciones. El resto de los resultados se especifica en la Tabla 1 y es integrado a la reconstrucción de los métodos de manufactura:

(1) La cuarcita fue la materia prima más efectiva para el desarrollo de las tareas de formatización de los instrumentos, debido a su dureza (7 en la escala de Mohs). Los raspadores y lascas elaborados con este tipo de roca fueron útiles para el raspado, ranurado y aserrado perimetral. Por otro lado, la ftanita no resultó adecuada para estas tareas, dado que los filos se fracturaron y astillaron. Por el contrario, la cuarcita no fue tan útil como pièce esquillè como la ftanita. La primera fue propensa a fracturarse al momento de separar las astillas por medio de fracturas controladas.

(2) Como es sabido, los huesos en estado seco son menos efectivos para su manufactura, debido a la pérdida de sus cualidades originales y a su menor capacidad para absorber el estrés (Evans 1973). Sin embargo, en todos los experimentos las formas base fueron obtenidas exitosamente. Por otro lado, no hubo diferencias entre los elementos del individuo juvenil y los de adultos al momento de la manufactura de los restos. Este aspecto podría haber sido esperable debido a que la estructura a nivel del hueso en las distintas categorías etarias no es igual (Lyman 1994). En el caso del elemento sumergido en agua, se registró que el ranurado, raspado y aserrado requirieron menos tiempo que para el resto de los elementos. 


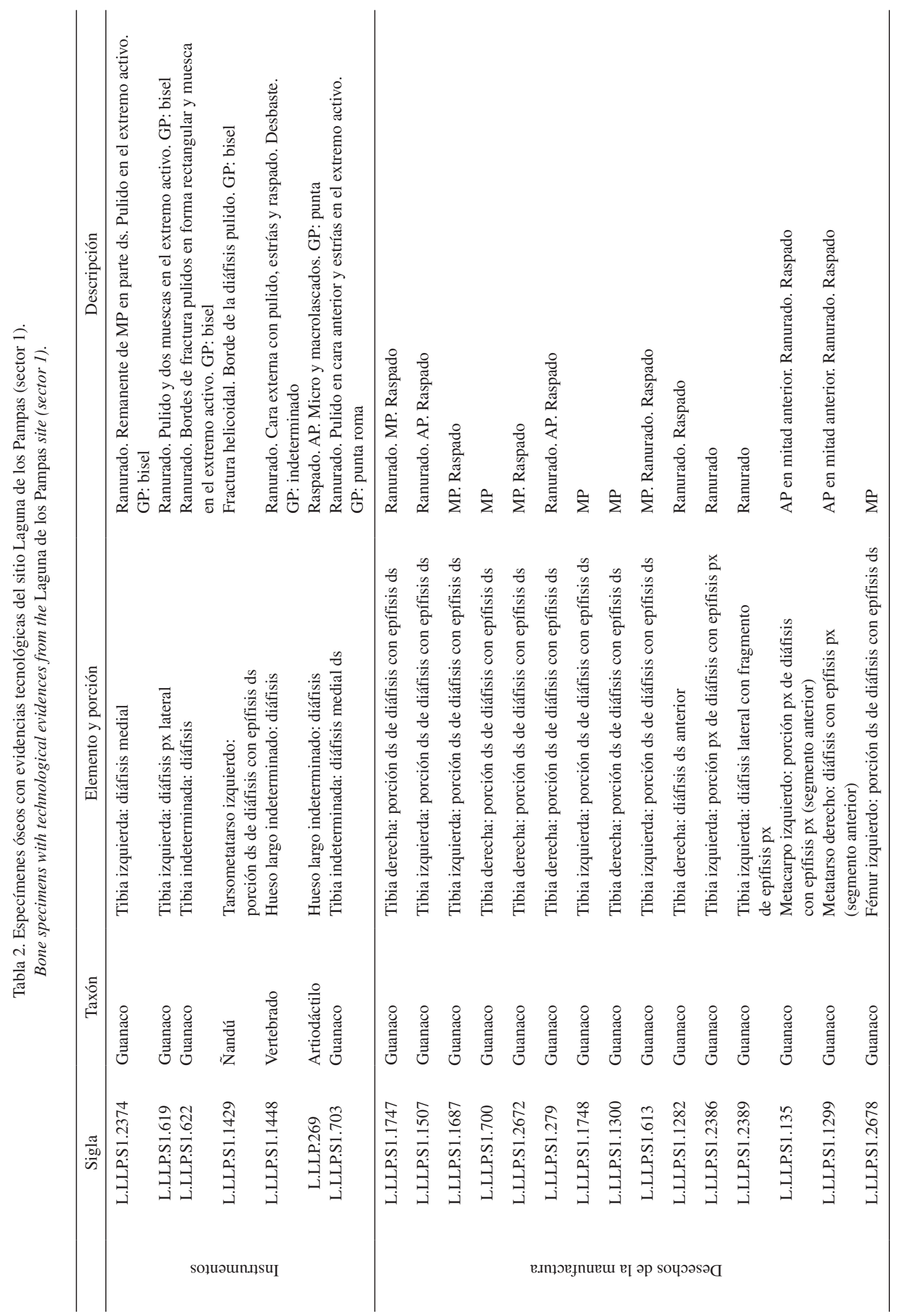


(3) En relación con el tiempo invertido, en todos los experimentos la obtención de las formas base tomó alrededor de dos horas y media. La excepción fue el elemento sumergido en agua, para el cual tomó dos horas.

(4) En el caso de la tibia se observó que el ranurado lateral y anterior hizo difícil la fractura por medio del uso de yunque. Esto es porque el rebote generado por el golpe bipolar produce una fractura en el lado contrario al que se golpea. En este elemento, en que los surcos no eran opuestos, se requirieron técnicas más controladas, como la profundización de los mismos hasta atravesar la diáfisis. En el caso de los metapodios, esto no fue necesario y el ranurado pudo realizarse en forma más superficial (Figura 5).

\section{Reconstrucción de la secuencia de manufactura de las formas base}

Considerando las evidencias arqueológicas y los materiales experimentales, se infirieron las secuencias descriptas a continuación para la obtención de las formas base. En el caso del metapodio: (1) raspado longitudinal alrededor de la metáfisis proximal, principalmente la cara anterior (raspadores de cuarcita); (2) ranurado de la cara lateral, desde el área de la metáfisis distal hasta el final de la epífisis proximal (filos naturales de lascas de cuarcita, puntas buriladas de cuarcita); (3) ranurado de la cara medial, desde el área de la metáfisis hasta el final de la epífisis proximal (filos naturales de lascas de cuarcita, puntas buriladas de cuarcita); (4) aserrado perimetral de la epífisis distal a la altura de la metáfisis (filos naturales de lascas de cuarcita); (5) aserrado de la cara anterior de la metáfisis proximal (filos naturales de lascas de cuarcita); (6) fractura preparada para remover la epífisis distal (percutor de granito); (7) separado de las astillas a través del uso de cuñas (pièce esquillè de ftanita, asta y percutor de cuarcita); (8) fractura preparada para remover la epífisis proximal anterior (percutor de granito). De esta secuencia se obtuvieron dos formas base (Figura 6a).

En el caso de la tibia: (1) raspado longitudinal alrededor del área de la metáfisis distal (raspadores de cuarcita), (2) ranurado en la cara lateral, siguiendo la incisión natural dada por la incisura fibularis en la porción distal; este surco continúa a lo largo de toda la diáfisis lateral y sigue hacia la cara posterior, terminando en una convergencia 


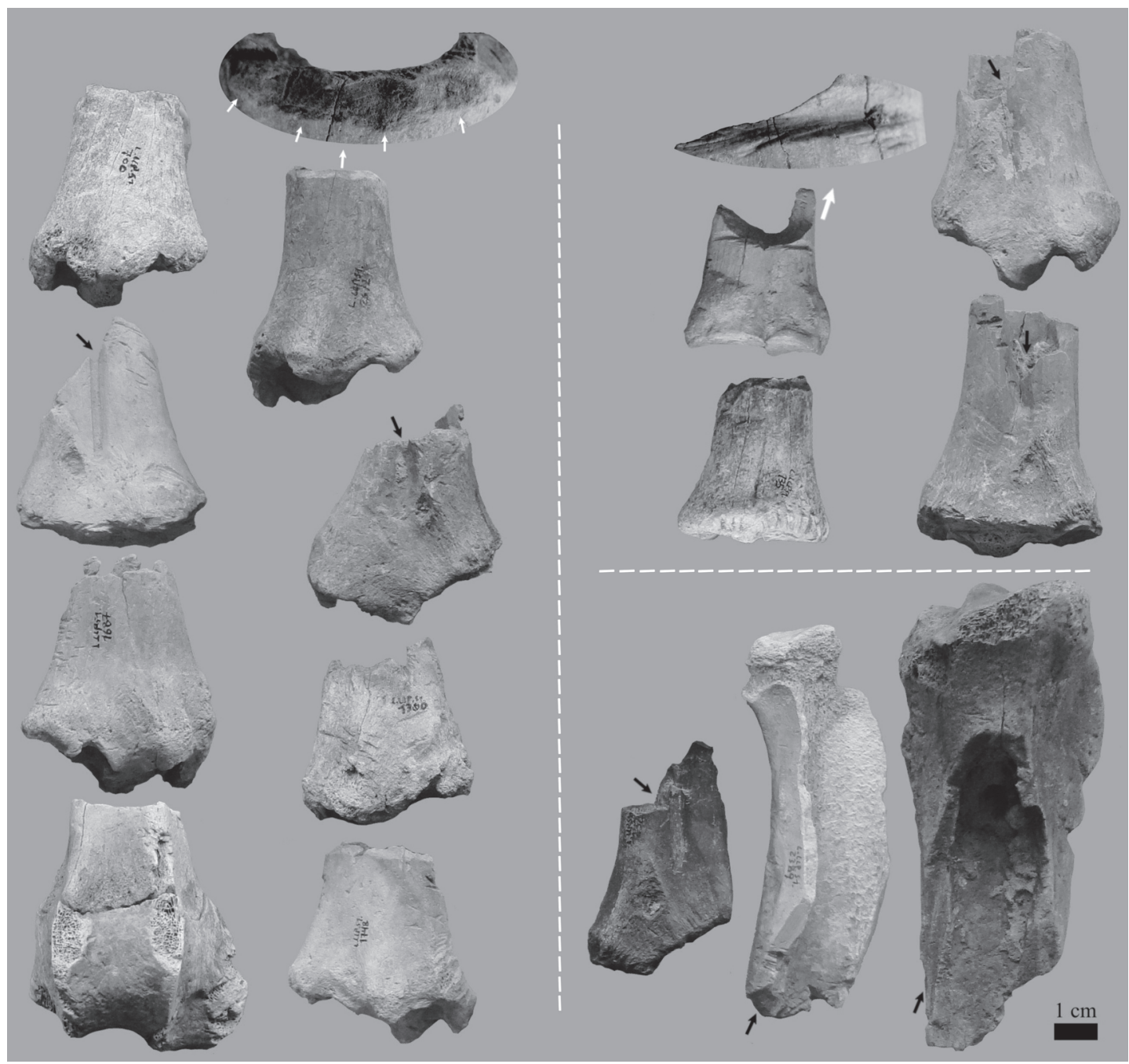

Figura 3. Desechos de manufactura de instrumentos óseos. A la izquierda se observan especímenes con marcado perimetral y arriba a la derecha especímenes con aserrado perimetral. Las flechas señalan remanentes de ranurado.

Debitage from bone tool manufacturing process. Left: transversally cut specimens; right: saw-cut specimens. Arrows indicate grooving.

con el surco anterior (filos naturales de lascas de cuarcita, puntas buriladas de cuarcita); (3) ranurado en la cara anterior; este surco va a lo largo de toda la cara anterior y hacia la cara lateral, terminando en la convergencia con el ranurado lateral (filos naturales de lascas de cuarcita, puntas buriladas de cuarcita); (4) aserrado perimetral en la metáfisis distal (filos naturales de lascas de cuarcita) o marcado perimetral de la misma (percutor de cuarcita); (5) fractura preparada para retirar la epífisis distal (percutor de granito); (6) separación de la diáfisis a través del uso de cuñas (pièce esquillè de ftanita, asta y percutor de cuarcita) o continuación de ranurado hasta atravesar la diáfisis (filos naturales de lascas de cuarcita, puntas buriladas de cuarcita). De esta secuencia se obtuvieron dos formas base (Figura 6b).

\section{Discusión y Consideraciones Finales}

A partir de este trabajo se reconstruyó la cadena operativa teórica de la manufactura de instrumentos confeccionados sobre tibias y metapodios en el sitio LLP. En relación con la tibia, dado que se recuperaron varios especímenes, las técnicas fueron inferidas principalmente sobre la base del registro arqueológico y sustentadas por los experimentos. Por otro lado, para el caso de los metapodios la secuencia fue hipotetizada de los dos únicos especímenes 


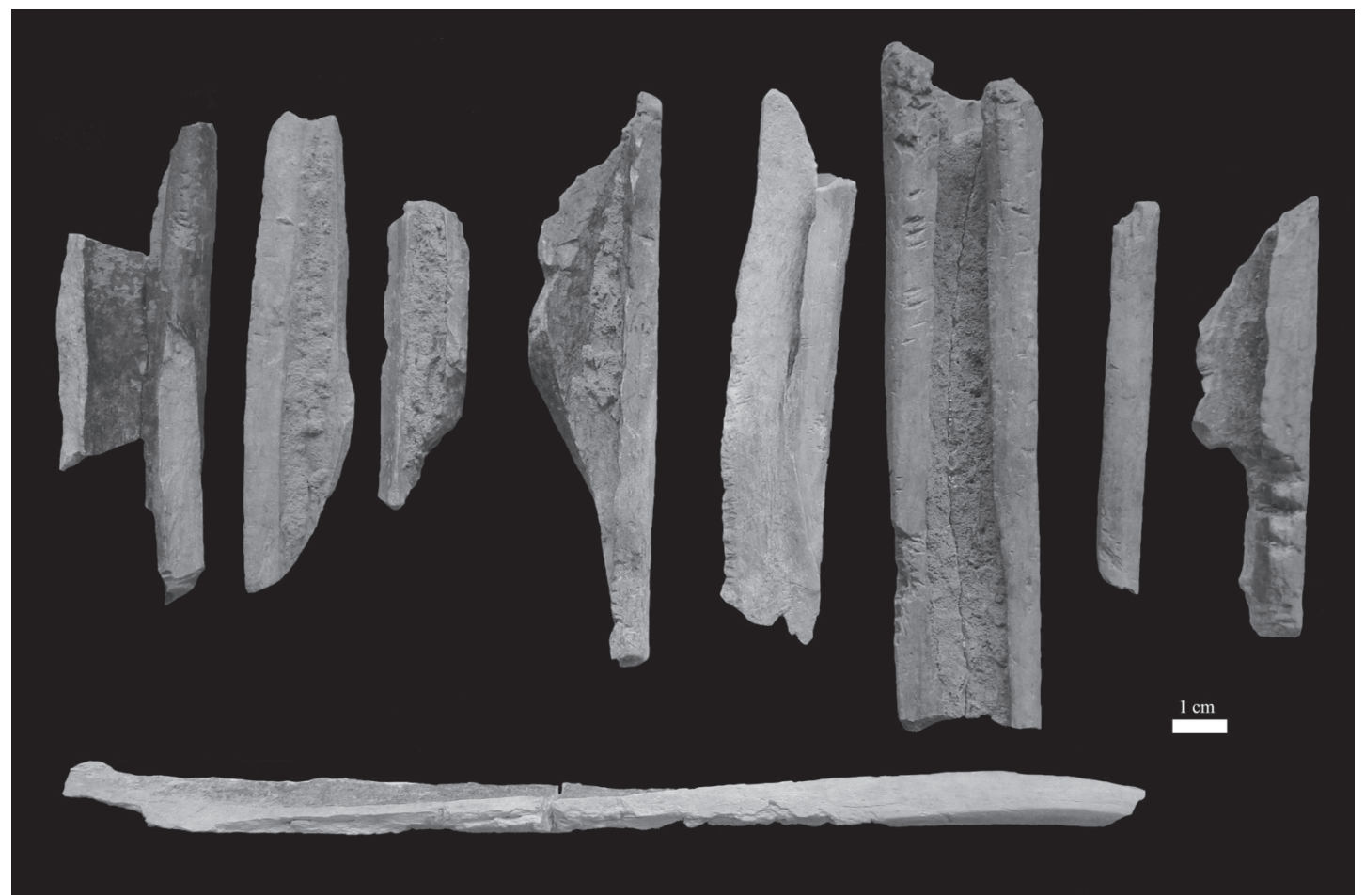

Figura 4. Diáfisis de huesos largos con evidencias tecnológicas correspondientes a formas base o instrumentos fracturados. Long bone shafts with technological evidence, corresponding to blanks or broken tools.

recuperados, así como a partir de los experimentos. $\mathrm{Al}$ respecto, en el caso del área Norte, Loponte y Buc (2012) identificaron la secuencia probable de manufactura para los punzones y puntas acanaladas, confeccionados sobre metapodios de venado de las pampas. Estos autores proponen que en el caso de los primeros, generalmente se descartó el extremo proximal de los metapodios en tanto que para el segundo grupo se desechó la epífisis distal. Respecto de los punzones, Loponte y Buc (2012) reconocieron dos técnicas de manufactura: por aserrado de la epífisis proximal y por fractura de la misma por percusión. Sin embargo, estos instrumentos se utilizan conservando la epífisis distal del mismo. Por otro lado, en un contexto similar, el sitio Laguna el Doce $(165 \mathrm{~km}$ al norte de LLP; Ávila 2011), se recuperaron instrumentos confeccionados sobre metapodios de guanaco que en su mayoría corresponden a biseles. Aunque aún no se ha identificado la secuencia de producción de los mismos, los instrumentos terminados no conservan la epífisis distal del elemento (Cornaglia Fernández y Buc 2013). Considerando estos aspectos, la cadena operativa inferida para el caso de
LLP podría tener algunas variaciones, en relación con las inferencias realizadas en otras áreas. Sin embargo, es difícil determinar los primeros pasos de la secuencia de producción del metapodio hasta tanto se tenga información del tipo de instrumento que se busca confeccionar a partir de este soporte.

En relación con los instrumentos, se identificaron los grupos morfológicos bisel (4), punta roma (1) y punta (1), en tanto que uno de ellos no pudo ser asignado a las categorías conocidas. El taxón soporte registrado fue el guanaco en la mayoría de los casos, así como en dos oportunidades se identificó artiodáctilo, que probablemente corresponda también a esta especie y un solo espécimen fue determinado como ñandú. Respecto de los elementos soporte, todos aquellos que pudieron ser identificados corresponden a tibias y, en el caso del ñandú, a un tibiotarso. Como se desprende de esto, la tibia fue seleccionada por sobre los metapodios como núcleo para la manufactura de instrumentos. Si bien podría ser el caso que los metapodios estuvieran vinculados a estrategias más conservadas y por eso sean más escasos, de todas formas sería esperable una mayor cantidad de 


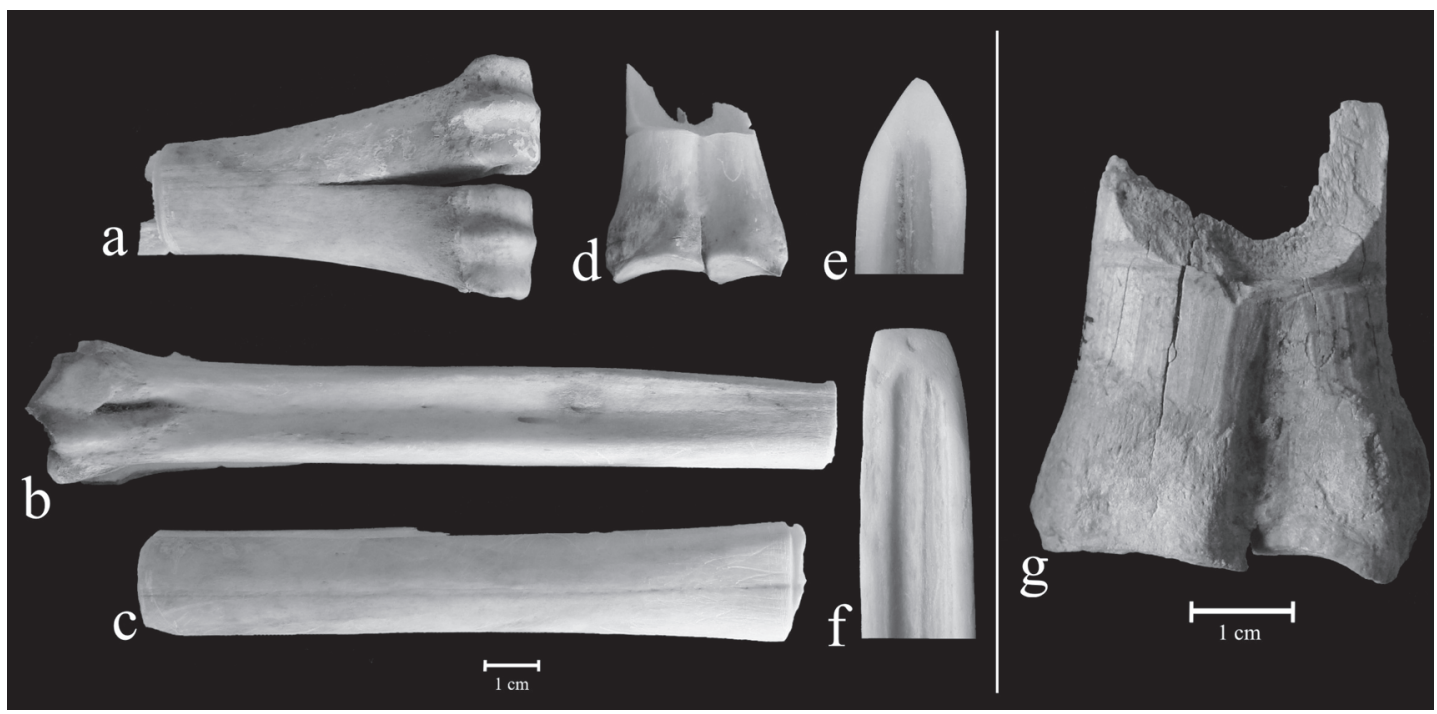

Figura 5. Ejemplo de uno de los experimentos: (a) y (d) desechos de la manufactura; (b) y (c) formas base obtenidas; (e) punta confeccionada sobre la diáfisis anterior; (f) bisel confeccionado sobre la diáfisis posterior; (g) desecho de manufactura arqueológico similar al obtenido a través de la experimentación mediante la técnica de splinter.

Example of one of the experiments: $(a)$ and $(d)$ are debitage; $(b)$ and $(c)$ obtained blanks; $(e)$ point made on the anterior shaft; (f) bevel end made on the posterior shaft; $(g)$ archaeological waste of debitage similar to that obtained through an experiment using the splinter technique.

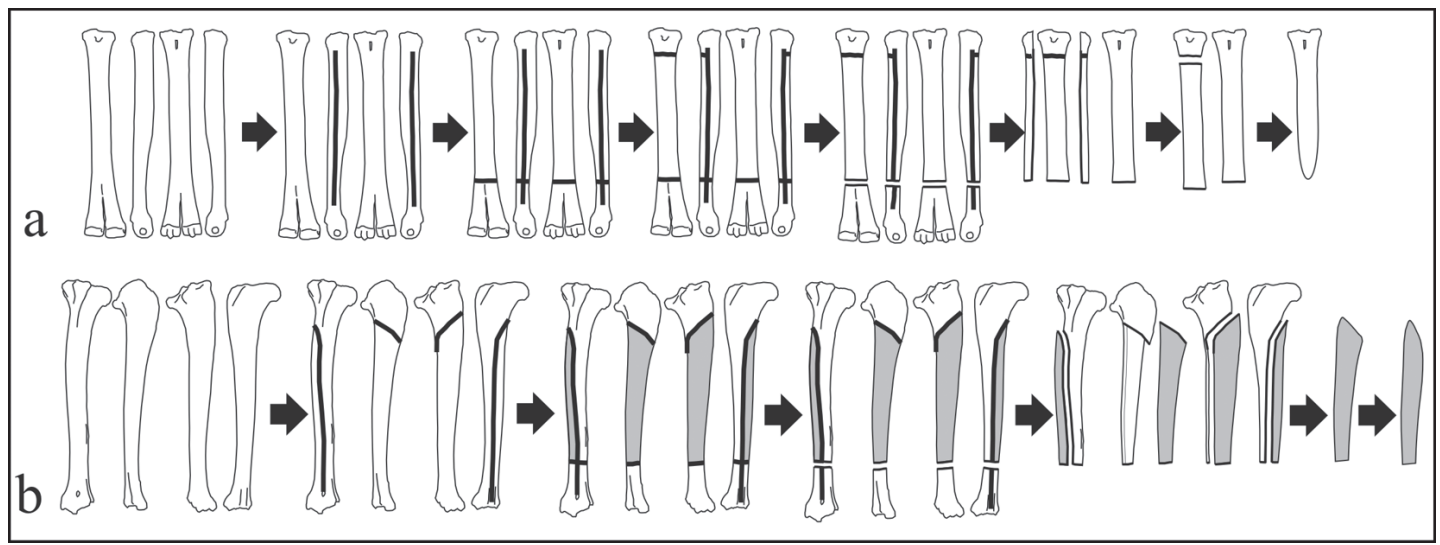

Figura 6. Cadena operativa de la obtención de formas base inferida a partir del registro arqueológico y experimental (a) metapodios; (b) tibia. En este último caso el sombreado corresponde al segmento más utilizado como forma base.

Chaîne opératoire of manufacture of blanks inferred from the archaeological and experimental record: (a) metapodials; (b) tibia. In the latter, the grey color indicates the shaft segment preferred for use as a blank.

desechos. En este punto es importante mencionar que ambos elementos están presentes en el registro zooarqueológico en frecuencias similares, con evidencias semejantes de porcentajes de fracturas antrópicas, posiblemente vinculadas a la subsistencia, con lo cual no se observa una representación diferencial en el transporte de unidades anatómicas o aprovechamiento, que pudieran condicionar el posterior uso de estas unidades para la tecnología ósea. En el sitio Laguna El Doce se recuperaron 10 instrumentos óseos. Al igual que en LLP, los biseles son abundantes y muestran una alta estandarización, pero contrariamente, los metapodios fueron los elementos soporte seleccionados por sobre el resto de las unidades anatómicas (Cornaglia Fernández y Buc 2013). Hasta el momento, la razón para la selección de tibias o metapodios para la manufactura de instrumentos no es clara. 
Si bien evidentemente depende de las propiedades de los huesos (Scheinsohn y Ferretti 1995), ambos elementos son igualmente adecuados para este fin, dado que presentan matrices derechas y elongadas.

Considerando la presencia de especímenes vinculados a distintas etapas de la cadena operativa representados en LLP, se propone que los instrumentos fueron confeccionados en el sitio para el caso de la tibia, en tanto que no es posible afirmar esto en el caso de los metapodios. Los restos óseos con evidencias tecnológicas contrastan con otros sitios del Sudeste de la región pampeana (principalmente las áreas de Tandilia e Interserrana) donde los mismos son escasos y los desechos de la manufactura están ausentes o no han sido identificados (Álvarez 2012). Mazzanti y Valverde (2001:167) reconstruyeron la cadena operativa de producción de artefactos sobre asta, metapodio de venado de las pampas y huesos largos no identificados provenientes del sitio Cueva Tixi (área de Tandilia). Para todos los casos los autores identifican que los instrumentos se confeccionaron sobre soportes obtenidos a partir de fracturas, por percusión de los elementos. Según Mazzanti y Valverde (2001), en el caso de los huesos largos, como los metapodios de venado de las pampas (seleccionados para la confección de punzones), la intención primaria de las fracturas habría sido la de la obtención de la médula ósea. La selección de los mismos como soporte habría sido una decisión posterior, tras la cual se realizó una formatización somera de las piezas. Por otra parte, los estudios preliminares realizados sobre especímenes con evidencias tecnológicas para el área Interserrana muestran una tendencia similar a la descripta por Mazzanti y Valverde (2001). Sin embargo, en dos sitios (La Toma y Paso Otero 4; Álvarez 2012) se identificaron especímenes con ranurado, lo cual indica que al menos no en todos los casos la selección de los soportes fue una consecuencia de las fracturas realizadas para el consumo. Contrariamente a las tendencias mencionadas para el Sudeste de la región pampeana, en el área Norte se observa una secuencia de producción más estandarizada, como fue expresado al inicio de la discusión para los punzones confeccionados sobre metapodios (Loponte y Buc 2012). Otro caso para el cual se cuenta con especímenes en distintas etapas de producción es el área del delta del río Paraná. Allí ha identificado la secuencia de elaboración para la manufactura de puntas cónicas sobre astas de cérvidos, la cual incluye procedimientos como el aserrado perimetral, ahuecado y desbaste de las cornamentas (Bonomo et al. 2009).

Dado que el guanaco fue el recurso central en la dieta de los cazadores-recolectores de la región, es lógico que los métodos de manufactura de los instrumentos estén vinculados a la adquisición de los subproductos de esta animal. Las técnicas de obtención de las formas base estuvieron relacionadas con el consumo de médula ósea, dada la importancia de este subproducto para estos grupos, como fue planteado en distintas oportunidades, debido a que la carne de guanaco es magra y el contenido medular aporta altos valores de grasa (Mengoni Goñalons 1999). También Mazzanti y Valverde (2001) mencionan que la adquisición de la médula de artiodáctilos estaría directamente vinculada con la obtención de los soportes en el sitio Cueva Tixi. Como fue mencionado, los restos óseos manufacturados de LLP contrastan con los de los sitios del Sudeste de la región pampeana, que son escasos y responden a una estrategia más expeditiva. El hecho de que las fuentes de materia prima lítica estén ausentes en el área (la más cercana se localiza $250 \mathrm{~km}$ al Sur) podría ser considerado como una posible causa para el uso sistemático de huesos como núcleos. Sin embargo, los restos líticos son abundantes en el sitio y a su vez se requiere de los mismos para manufacturar los primeros. También es importante tener en cuenta que el conjunto lítico de LLP no muestra diferencias significativas con otros sitios de la región pampeana donde los afloramientos rocosos son más cercanos, con excepción del menor tamaño de los artefactos (Politis et al. 2012). En este sentido, la opción más probable es que la tecnología ósea esté vinculada con actividades específicas que aún no han sido identificadas. Los análisis funcionales microscópicos que serán desarrollados a futuro, a partir de la ampliación de la muestra experimental, ayudarán a clarificar el uso dado a estos instrumentos, aunque es posible que los resultados sean sesgados por las modificaciones postdepositacionales, como la presencia de carbonato de calcio en algunos especímenes. Al respecto, los estudios llevados a cabo en el sitio El Doce indican la utilización de al menos dos de los biseles en la manufactura de artefactos líticos (Cornaglia Fernández y Buc 2013).

En conclusión, este estudio permitió inferir las técnicas de manufactura a partir de evidencias arqueológicas y experimentales. Los resultados indicaron una estandarización en la producción de 
la tecnología ósea de los grupos cazadores recolectores que habitaron el Oeste de la Pampa Húmeda, al menos durante el Holoceno Medio, tanto en el sitio LLP (este trabajo) como en El Doce (Cornaglia Fernández y Buc 2013). Esto se diferencia del Sudeste de la región pampeana, en la cual la tecnología ósea se orientó hacia el extremo más expeditivo (Clase 2), si bien en momentos tempranos hubo una formatización más elaborada de algunos artefactos (Álvarez 2012; Mazzanti y Valverde 2001). En el caso del área Norte y la Depresión del Salado, las diferencias más notables están vinculadas con las adaptaciones fluviales de estos grupos (Politis y Leon 2010), lo cual se ve reflejado en los taxones soporte (p.ej., espinas de peces), así como en el caso de algunos grupos morfológicos altamente estandarizados, como las puntas de arpón sobre asta (Acosta et al. 2010; Bonomo et al. 2009; Buc 2007; Pérez Jimeno 2004; entre otros). En suma, en el área Oeste se observan características particulares de la tecnología ósea, que diferencian a la misma de otras aledañas y aportan nueva información, poniendo de manifiesto la estandarización en la manufactura de instrumentos, centralizada principalmente en tibias de guanaco, sobre las cuales se han confeccionado mayoritariamente instrumentos en bisel.

Agradecimientos: A Eva David, por sus comentarios y sugerencias a este manuscrito. A quienes participaron en las tareas de campo y el desarrollo de los experimentos: Pablo Messineo, Mariela González, Gustavo Politis, Manuel Carrera, Ana Paula Alcaraz, Yamile Infantas y Florencia Santos. Especialmente a Paula Barros y a Cristián Kaufmann, quien proveyó las unidades anatómicas de guanaco. A las autoridades de Lincoln/Martínez de Hoz, Ramón Coria y los dueños y administradores de los campos de LLP; Sergio Leguizamón, Juan Rodríguez, Nicolás Giaccone y Luis Musso. A dos evaluadores anónimos quienes con sus comentarios y sugerencias contribuyeron a mejorar sustancialmente este manuscrito.

\section{Referencias Citadas}

Acosta, A. 2000. Huellas de corte relacionadas con la manufactura de artefactos óseos en el nordeste de la provincia de Buenos Aires. Relaciones de la Sociedad Argentina de Antropología XXV:159-178.

- - - 2005. Zooarqueología de Cazadores-Recolectores del Extremo Nororiental de la Provincia de Buenos Aires (Humedal del río Paraná Inferior, Región Pampeana, Argentina). Tesis para optar al título de Doctor en Ciencias Naturales. Facultad de Ciencias Sociales y Museo. Universidad Nacional de La Plata, La Plata.

Acosta, A., N. Buc y D. Pau 2011. Huellas de aserrado perimetral sobre restos óseos humanos. El caso del sitio Cerro Lutz, provincia de Entre Ríos. Comechingonia 15(1):155-169.

Acosta, A., S. Escudero, M.R. Feuillet Terzaghi, D. Loponte y L. Pérez Jimeno 2010. Conectando registros: variabilidad arqueológica en la Cuenca del Paraná. En Mamül Mapu: Pasado y Presente desde la Arqueología Pampeana Tomo 2, editado por M. Berón, L. Luna, M. Bonomo, C. Montalvo, C. Aranda y M. Carrera Aizpitarte, pp. 17-28. Libros del Espinillo, Buenos Aires.

Álvarez, M.C. 2012. Análisis Zooarqueológicos en el Sudeste de la Región Pampeana. Patrones de Subsistencia Durante el Holoceno Tardío. Tesis para optar al título de Doctora en Arqueología. Facultad de Ciencias Sociales, Universidad Nacional del Centro de la Provincia de Buenos Aires, Olavarría.

Averbouh, A. 2000. Technologie de la Matière Osseuse Travaillée et Implications Palethnologiques: l'Exemple des Chaînes d'Exploitation du Bois de Cervidé chez les Magdaléniens des Pyrénées. Tesis Doctoral. uFr d'Histoire de l'art et l'archéologie, Université de Paris I Panthéon-Sorbonne, París.
Avila, J.D. 2011. Resultados de los fechados radiocarbónicos del sitio Laguna El Doce, departamento de General López, provincia de Santa Fe. Relaciones de la Sociedad Argentina de Antropología XXXVI:337-343.

Behrensmeyer, A. 1978. Taphonomic and ecologic information from bone weathering. Paleobiology 4:150-162.

Binford, L. 1981. Bones: Ancient Men and Modern Myths. Academic Press, New York.

Bonomo, M. 2013. Reanálisis de la colección de Samuel Lothrop procedente del delta del Paraná. Relaciones de la Sociedad Argentina de Antropología XXXVIII (1):169-198.

Bonomo, M., I. Capdepont y A. Matarrese 2009. Alcances en el estudio de colecciones. Los materiales arqueológicos del delta del río Paraná depositados en el museo de La Plata (Argentina). Arqueología Sudamericana 5:68-101.

Bonomo, M., G. Politis y C. Gianotti 2011. Montículos, jerarquía social y horticultura en las sociedades indígenas del delta del río Paraná (Argentina). Latin American Antiquity 22:297-333.

Borao Álvarez, M. 2013. Identificación de los elementos tecnológicos del proceso de producción de industria ósea en el registro faunístico. Aplicación a la secuencia del magdaleniense superior de la Cova de les Cendres (Teulada-Moraira, alicante). En Animals i Arqueologia, editado por A. Sanchis Serra y J.L. Pascual Benito, pp. 109-132. Museu de Prehistòria de València, Valencia.

Borella, F. y N. Buc 2009. Ópticas y ópticos. Una aproximación a la tecnología ósea en la Bahía de San Antonio (Río Negro), Argentina. En Arqueología de la Patagonia. Una Mirada desde el Último Confín, editado por M. Salemme, F. Santiago, 
M. Álvarez, E. Piana, M. Vázquez y S. Mansur, pp. 421-432. Editorial Utopías, Ushuaia.

Borrero, L.A. y F. Borella 2010. Harpoons and travellers: Fuegian ethnographic collections and the recent archaeological record. Before Farming 1:1-14.

Buc, N. 2011. Experimental series and use-wear in bone tools. Journal of Archaeological Science 38:546-557.

- - - - 2007. Ser o no ser: arpones y "arpones b" en el humedal del Paraná inferior. En Arqueología en las Pampas. Tomo 1, editado por C. C. Bayón, A. Pupio, M. I. González, N. Flegenheimer y M. Frère, pp. 325-342. Sociedad Argentina de Antropología, Buenos Aires.

Buc, N. y L. Pérez Jimeno 2010. Puntas para la comparación de la tecnología ósea en el Paraná Interior y Medio. En Zooarqueología a Principios del Siglo XXI. Aportes Teóricos, Metodológicos y Casos de Estudio, editado por M. Gutiérrez, M. De Nigris, P. Fernández, M. Giardina, A. Gil, A. Izeta, G. Neme y H. Yacobaccio, pp. 439-458. Ediciones del Espinillo, Buenos Aires.

Buc, N. y R. Silvestre 2006. Funcionalidad y complementariedad de los conjuntos líticos y óseos en el humedal del nordeste de la provincia de Buenos Aires: Anahí, un caso de estudio. Intersecciones en Antropología 7:129-146.

Caggiano, M.A. 1984. Prehistoria del noreste Argentino, sus vinculaciones con la República Oriental del Uruguay y sur de Brasil. Pesquisas: Antropología 38:1-109.

Campana, D.V. 1980. An Analysis of the Use-Wear Patterns on Natufian and Protoneolithic Bone Implements. Tesis Doctoral, Columbia University, New York.

Camps-Fabrer, H. y A. D’Anna 1977. Fabrication expérimentale d'outils à partir de métapodes de mouton et de tibias de lapin. CNRS Deuxième Colloque International sur l'Industrie de l'os dans la Préhistoire, Méthodologie Appliquée a L'Industrie de l'os Préhistorique: 311-323.

Choyke, A.M. 1997. The bone tool manufacturing continuum. Anthropozoologica 25-26:65-71.

Cornaglia Fernández, J. y N. Buc 2013. Evidence of bone technology on the Santa Fe's Pampa lagoons. The Laguna El Doce Site (Santa Fe province, Argentina). En From these Bare Bones: Raw Materials and the study of Worked Osseous Objects, editado por A. Choyke y S. O'Connor, pp. 109-115. Oxbow Books, Oxford.

David, E. 2004. Fiche transformation des matières dures d'origine animale dans le mésolithique ancient d'Europe du Nord. Societé Préhistorique Française XI:113-149.

- - - 2007. Technology on bone and antler industries: a relevan methodology for characterizing early post-glacial societies $\left(9^{\text {th }}\right.$ - $8^{\text {th }}$ millennium BC). En Bones as Tools: Current Methods and Interpretations in Worked Bone Studies, editado por C. Gates St-Pierre y R.B. Walker, pp. 35-50. BAR International Series 1622, Oxford.

Demars, P.Y. y P. Laurent 1989. Types d'outils lithiques du Paleolithique superieure en Europe. Cahiers du Quaternaire 14:1-178.

Emery, K. 2008. Techniques of ancient Maya bone working: evidence form a classic Maya deposit. Latin American Antiquity 19:204-221.
Evans, F.G. 1973. Mechanical Properties of Bone. Charles C. Thomas, Publisher, Springfield.

González, M.I. 2005. Arqueología de Alfareros, Cazadores y Pescadores Pampeanos. Sociedad Argentina de Antropología, Buenos Aires.

González, M.I., M. Lanza y C. Tambussi 2004. Registro arquefaunístico de aves en ambientes lagunares, Curso inferior del Salado, Buenos Aires. En La Región Pampeana, su Pasado Arqueológico, editado por C. Gradín y F. Oliva, pp. 337-346. Laborde Editor, Buenos Aires.

Goutas, N. 2004. Caractérisation et évolution du Gravettien en France par l'approche Techno-éco nomique des Industries en Matières Dures Animales (Étude de six Gisements du SudOuest). Tesis Doctoral. uFr d'Histoire de l'art et l'archéologie, Paris i-Panthéon-Sorbonne, París.

Grayson, D. 1984. Quantitative Zooarchaeology: Topics in the Analysis of Archaeological Faunas. Academic Press, Orlando.

Gutiérrez, M. 2006. Efectos, agentes y procesos tafonómicos en el área Interserrana Bonaerense. Relaciones de la Sociedad Argentina de Antropología XXXI:201-228.

Gutiérrez, M.A. y C.A. Kaufmann 2007. Criteria for the identification of formation processes in guanaco (Lama guanicoe) bone assemblages in fluvial-lacustrine environnments. Journal of Taphonomy 5:151-176.

Hajduk, A. y M. Lezcano 2005. Un "nuevo-viejo" integrante del elenco de instrumentos óseos de Patagonia: los machacadores óseos. Magallania 33:63-80.

Haynes, G. 1980. Evidence of carnivore gnawing on Pleistocene and recent mammalian bones. Paleobiology 6:341-351.

- - - 2000. Mammoths, measured time, and mistaken identities. Radiocarbon 42:257-269.

Johnson, E. 1985. Current developments in bone technology. En Advances in Archaeological Method and Theory 8, editado por M.B. Schiffer, pp. 157-235. Academic Press, New York.

Johnson, E., G. Politis y M. Gutiérrez 2000. Early Holocene bone technology at the La Olla site, Atlantic coast of the Argentine pampas. Journal of Archaeological Science 27:463-477.

Kaufmann, C. 2009. Estructura de Edad y Sexo en Guanaco. Estudios Actualísticos en Pampa y Patagonia. Sociedad Argentina de Antropología, Buenos Aires.

Lemmonier, P. 1976. L'étude des systémes techniques, une urgence en technologie culturelle. Techniques et Cultures 1:100-151.

Loponte, D. y N. Buc 2012. Don't smash those bones! Anatomical representation and bone tools manufacture in the Pampean region (Argentina, South America). En Bones for Tools, Tools for Bones: The Interrelationship of Lithic and Bone Raw Materials, editado por K. Seetah y M. Gravina. McDonald Institute for Archaeological Research, University of Cambridge, en prensa.

Lothrop, S. 1932. Indians of the Paraná delta, Argentina. Annals of the New York Academy of Science 32:77-232.

Lyman, R.L. 1994. Vertebrate Taphonomy. Cambridge Manuals in Archaeology. Cambridge University Press, Cambridge.

Mazzanti, D. y F. Valverde 2001. Artefactos sobre hueso, asta y valva. En Cueva Tixi: Cazadores y Recolectores de las Sierras 
de Tandilia Oriental, editado por D. Mazzanti y C. Quintana, pp. 157-180. Laboratorio de Arqueología, Universidad Nacional de Mar del Plata, Mar del Plata.

Mengoni Goñalons, G. 1999. Cazadores de Guanaco de la Estepa Patagónica. Sociedad Argentina de Antropología, Buenos Aires.

Messineo, P.G. y N. Pal 2011. Techno-morphological and usewear analysis on lithic and bone tools from Campo Laborde site (pampean region, Argentina). Current Research in the Pleistocene 28:110-112.

Muñoz, A. S. y J. B. Belardi 1998. El marcado perimetral en los huesos largos de guanaco de Cañadón Leona (Colección Junius Bird): implicaciones arqueofaunísticas para Patagonia Meridional. Anales del Instituto de la Patagonia 26:107-118.

Newcomer, M. 1974. Study and replication of bone tools from Ksar Akil (Lebanon). World Archaeology 2:138-153.

Olsen, S. 1980. Bone artifacts from Kinshba Ruin: their manufacture and use. The Kiva 46 (1-2):39-67.

Olsen, S. y P. Shipman 1988. Surface modification on bone: trampling versus butchery. Journal of Archaeological Science 15:535-553.

Orquera, L.A. y E.L. Piana 1999. La Vida Material y Social de los Yámana. Eudeba, Instituto Fueguino de Investigaciones Científicas, Buenos Aires.

Pacheco, V., A. Altamirano y E. Guerra 1979. Guía Osteológica de Camélidos Sudamericanos. Gabinete de Arqueología, Serie de Investigaciones, Universidad Nacional Mayor de San Marcos.

Paunero, R., M. Paunero y D. Ramos 2010. Artefactos óseos en componentes del Pleistoceno final de las localidades La María y Cerro Tres Tetas, Santa Cruz, Argentina. En Zooarqueología a Principios del Siglo XXI. Aportes Teóricos, Metodológicos y Casos de Estudio, editado por M. Gutiérrez, M. De Nigris, P. Fernández, M. Giardina, A. Gil, A. Izeta, G. Neme y H. Yacobaccio, pp. 459-466. Ediciones del Espinillo, Buenos Aires.

Pelegrin, J., C. Karlin y P. Bodu 1988. "Chaînes opératoires": un outil pour les préhistoriens. En Technologie Préhistorique (Notes et Monographies Techniques du CRA $N^{o} 25$ ), editado por J. Tixier, pp. 55-62. Ediciones del CNRS, París.

Pérez Jimeno, L. 2004. Análisis comparativo de dos conjuntos de artefactos óseos procedentes de la llanura aluvial del Paraná y la pampa bonaerense. En Aproximaciones Contemporáneas a la Arqueología Pampeana, Perspectivas Teóricas, Metodológicas, Analíticas y Casos de Estudio, editado por G. Martínez, M. Gutiérrez, R. Curtoni, M. Berón y P. Madrid, pp. 319-333. Universidad Nacional del Centro de la Provincia de Buenos Aires, Olavarría.

-- - 2007. Investigaciones Arqueológicas en el Sector Septentrional de la Llanura Aluvial del Paraná, Margen Santafesina. La Variabilidad del Registro Arqueológico. Tesis Doctoral, Facultad de Ciencias Naturales y Museo. Universidad Nacional de La Plata, La Plata.

Pérez Jimeno, L., M.R. Feuillet Terzaghi y S. Escudero 2010. Evidencias de tecnología ósea en la llanura aluvial del río Paraná medio e inferior-margen santafesina. En Zooarqueología a Principios del Siglo XXI. Aportes Teóricos, Metodológicos y Casos de Estudio, editado por M. Gutiérrez, M. De Nigris, P. Fernández, M. Giardina, A. Gil, A. Izeta, G. Neme y H. Yacobaccio, pp. 467-476. Ediciones del Espinillo, Buenos Aires.
Pickering, M. 1980. Bone tools from photographs in the Donald F. Thompson collection, National Museum of Victoria. The Artifact 5 (1-2):93-97.

Politis, G. 1988. Paradigmas, modelos y métodos en la arqueología de la Pampa Bonaerense. En Arqueología Contemporánea Argentina, pp. 59-107. Edición Búsqueda, Buenos Aires.

Politis, G., M. Bonomo, C. Castiñeira y A. Blasi 2011. Archaeology of the upper Delta of the Paraná River (Argentina): mound construction and anthropic landscapes in the Los Tres Cerros locality. Quaternary International 245:74-88.

Politis, G. y C. Leon 2010. Patrones adaptativos de los cazadores recolectores-pescadores de la margen occidental del Paraná inferior-Plata. En Arqueología de Cazadores Recolectores en la Cuenca del Plata, editado por G. Cocco y M.R. Feuillet Terzaghi; pp. 63-86. Centro de Estudios Hispanoamericanos, Santa Fé.

Politis, G., P. Messineo, M.E. González, M.C. Álvarez y C. Favier Dubois 2012. Primeros resultados de las investigaciones en el sitio Laguna de los Pampas (partido de Lincoln, provincia de Buenos Aires). Relaciones de la Sociedad Argentina de Antropología XXXVII:463-472.

Provenzano, N. 2004. Fiche terminologie du travail des matières osseuses, du Paléolithique aux Âges des Métaux. En Fiches Typologiques de l'Industrie Osseuse Préhistorique, CahierXI: Matières et Techniches. Commission de nomenclature sur l'industrie de l'os préhistorique, Société Préhistorique Française, París.

Rodríguez, J.A. 2001. Nordeste prehispánico. En Historia Argentina Prehispánica, editado por E. Berberián y A. Nielsen, pp. 693-736. Brujas, Córdoba.

Russell, N. 2006. Çatalhöyük worked bone. En Changing Materialities at Çatalhöyük: Reports from the 1995-99 Seasons, editado por H. Hodder, pp. 339-367. McDonald Institute for Archaeological Research, Cambridge.

Scheinsohn, V. 2010. Hearts and Bones: Bone Raw Material Exploitation in Tierra del Fuego. BAR International Series 2024, Oxford.

Scheinsohn, V. y J.L. Ferretti 1995 . The mechanical properties of bones materials in relation to the design and function of prehistoric tools from Tierra del Fuego, Argentina. Journal of Archaeological Science 22:711-717.

Serrano, A. 1950. Los Primitivos Habitantes de Entre Ríos. Biblioteca Entrerriana “General Perón”, Ministerio de Educación, Provincia de Entre Ríos, Paraná.

Shipman, P. 1981. Life History of a Fossil. Harvard University Press, Cambridge.

Tejero, J.M. 2010. La Explotación de las Materias Duras Animales en el Paleolítico Superior Inicial. Aproximación Tecno-Económica a las Producciones Auriñacienses en la Península Ibérica. Tesis Doctoral. Departamento de Prehistoria y Arqueología. UNED, Madrid.

Torres, L.M. 1911. Los Primitivos Habitantes del Delta del Río Paraná. Biblioteca Centenaria 4, Universidad Nacional de La Plata, Buenos Aires.

Yesner, D.R. y R. Bonnichsen 1979. Caribou metapodial shaft splinter technology. Journal of Archaeological Science 6:303-308. 


\section{Nota}

1 En este trabajo se consideran las divisiones realizadas por Politis (1988) para la región pampeana sobre la base de tradiciones de investigación consensuadas. Para la subregión
Pampa Húmeda se consideran las áreas Norte, Depresión del Salado, Interserrana, Tandilia, Ventania, Oeste y Sur (Figura 1). 